\title{
Growth Hormone (GH) Improvement of Ovarian Responses and Pregnancy Outcome in Poor Ovarian Responders: A Randomized Study
}

\author{
Leyli Safdarian', Marzieh Aghahosseini', Ashraf Alyasin', Atefeh \\ Samaei-Nouroozi ${ }^{1 *}$, Sahar Rashidi ${ }^{1}$, Maryam Shabani-Nashtaei ${ }^{2}$, Ayda Najafian ${ }^{1}$, \\ Parvane Lak ${ }^{1}$
}

\begin{abstract}
Recent evidence has emphasized growth hormone benefits in increasing the ovarian response and improving the pregnancy rate in poor responders (POR), caused by aging, ovarian surgery, chemotherapy and other reasons, undergoing IVF/ICSI. The most important factor in the treatment of POR patients is the quality and quantity of oocytes following ovarian stimulation; thus, efforts should be made to provide opportunities for young patients to improve their fertility and ovarian responses. The use of GH in these patients may offer a promising aid to successful fertility.In the present single-blinded clinical trial, POR patients were randomly assigned to receive one of three regimens: (A) Gonadotropin, a GnRH antagonist and GH from the eighth day of the cycle for about 5 days $(n=34)$; (B) Gonadotropin, a GnRH antagonist and $\mathrm{GH}$ from the third day of the previous cycle for about 20 days $(\mathrm{n}=32)$; and (C) Gonadotropin, a GnRH antagonist, and a placebo from the eight day of the cycle for about 5 days $(n=26)$. Oocyte quality and pregnancy rates were compared across the three groups. A significantly lower number of collected oocytes, MII oocytes, fertilized oocytes, transferred embryos, and clinical pregnancy rate in the placebo group was noted as compared to the two experimental groups receiving GH. Live clinical pregnancies in B group were significantly greater than in the other groups. Our results together indicate that GH may play an important role in recruitment of dominant follicles and enhance follicular survival and the cell proliferation leading to high- quality embryos. Accordingly, administration of GH can considerably elevate the ovarian response in patients with POR planned to undergo IVF.
\end{abstract}

Keywords: Growth hormone- poor ovarian response- pregnancy- outcome- in vitro fertilization

Asian Pac J Cancer Prev, 20 (7), 2033-2037

\section{Introduction}

The trend shifting towards delayed child bearing brought up the concept of ovarian ageing and many challenges against treatment in infertile patient that are called poor responder women. Poor ovarian response is mostly genetically determined, but acquired modifiable factors like aging, or ovarian surgery, Chemotherapy and radiotherapy are effective in improvement of survival rates of patients with cancer, too. Infertility is an important clinically-recognizable squeal in patients that can affect the sufferers' quality of life. Cryopreservation and transplantation of ovarian tissue are the best options for fertility preservation in adult patients with cancer (Sooyoung et al., 2018). However, if these patients refer several years after chemotherapy, these treatments are not effective and ovulation induction is inevitable.

Despite increasing advances in the use of fertility techniques such as IVF, a significant proportion of patients are still poor responders (PORs) in terms of oocyte recovery and pregnancy rates. In some reports, the incidence of such cases surpasses a third of the total subjects planned for IVF (Oehninger, 2011). The management of these PORs to reserve ovarian and fertility constitutes a formidable challenge among physicians and researchers. Various studies have been performed to increase the ovarian response and improve the outcome of pregnancy in patients undergoing IVF/ICSI (Venetis et al., 2010). One of the main strategies for stimulating the ovarian function is the use of gonadotropin releasing hormone (GnRH) agonists (Hamdine et al., 2015). However, the use of GnRH agonists in PORs has a lot of limitations, since it leads to inhibition of ovarian function and response that ultimately increases the dose and duration of gonadotropin use, causing early LH secretion and increase of IVF failure rate (Kucuk et al., 2008). 
Another stimulant protocol is the use of growth hormone (GH) that stimulates granulose cells and increases gonadotropin secretion investigated in both human and animal models. GH induces the production and secretion of IGF-1, which plays an important role in ovarian estrogen production (Mendoza et al., 2002). However, there is no consensus on the efficacy of GH. Moreover, for over 20 years, the positive effect of $\mathrm{GH}$ on improving the outcomes of pregnancy has been recognized in PORs; however, in recent years, the widespread use of GH has been limited as an ovarian stimulation protocol due to its high cost. Nowadays, with the increasing competition of pharmaceutical companies and breaking the exclusive circle of GH production and lowering its price, the field for the re-development of the $\mathrm{GH}$ has been opened as a successful protocol in stimulating ovarian potential (Tesarik et al., 2002). The exact mechanism of GH effect in improving the outcomes of pregnancy in IVF cycles is still unknown, but the GH seems to affect the process of steroid production in the ovary, leading to follicular development. Studies have shown that maturation and evolution of oocytes have been impaired or severely reduced by inhibiting the GH receptor in animal model (Lucy, 2011). The benefits of GH have been also revealed in human models. In a recent systematic review and meta-analysis, the addition of GH to gonadotropins has been beneficial in ovarian stimulation of POR group treated by IVF (Lucy, 2000; Kolibianakis et al., 2009). However, because of employing small examined human samples, further clinical trials are warranted to prove or disprove this finding. The present study aimed to assess the role of $\mathrm{GH}$ in improvement of pregnancy outcome in PORs.

\section{Materials and Methods}

\section{Study population}

The present study was a single-blinded clinical trial in which 105 PORs, who referred to Dr Shariati Hospital between May 2016 and September 2017 in order to treat infertility, were assessed initially and then randomly assigned to three groups. The current study was approved by Tehran University Medical Ethics Committee (IR.TUMS.VCR.REC. 1395.1186) and it was in accordance with the Helsinki Declaration of 1975, revised in 1983. This study was also registered in Iranian Registry of Clinical Trials (IRCT20140818842N14). The randomization was done using a computerized random sampling table, and patients' blinding was also considered.

As shown in Figure 1, one patient in group A (due to fertilization failure), three patients in group B (due to fertilization failure), and nine patients in group $C$ (due to oocytes retrieval failure and fertilization failure) were excluded. Finally, 34, 32 and 26 patients were analyzed in group A, B, and C, respectively. The inclusion criteria were being POR (due to aging, ovarian surgery, chemotherapy and unknown reasons) based on the criteria provided by the European Institute for Embryology and Infertility in 2011 as follows (having at least two of the three following criteria): 1) age over 40 years, 2) the evidences of POR as having a maximum of three oocyte following induction protocol), and 3) low ovarian reserve score (AFC less than 5-7, AMH less than $0.5-1.1 \mathrm{ng} / \mathrm{ml}$ ). Women with high FSH levels (more than $20 \mathrm{IU} / \mathrm{L}$ ), a history of infertility due to known non-POR causes such as azoospermia, 3 diabetes (type one or two), and those who were not willing to participant in the study were excluded.

\section{Study interventions}

The conditions of this clinical trial were evaluated based on the Consolidated Standards of Reporting Trials (CONSORT) guideline. The patients in all groups received gonadotropine (Gonal-f300 to $450 \mathrm{IU} /$ day, subcutaneously, based on age, AFC, and the level of AMH) plus GnRH antagonist (Cetrotide, $0.25 \mathrm{mg} /$ day, subcutaneously, after production of $14 \mathrm{~mm}$ follicles until $\mathrm{HCG}$ injection) from the third day of their cycle. In addition to common regimens, group A received GH (Somatropin, $2.5 \mathrm{mg} /$ day, subcutaneously from the eighth day of the cycle until the injection of HCG), group B received GH (Somatropin, $0.1 \mathrm{mg} /$ day, subcutaneously from the third day of the previous cycle), and group $\mathrm{C}$ received placebo (normal saline, $0.1 \mathrm{mg} /$ day, subcutaneously) from the eighth day of the cycle until the injection of HCG). Growth follicles were monitored on the eighth day following Gonadotropine (Gonal-f) administration. If a follicle more than $18 \mathrm{~mm}$ was revealed, the trigger of ovulation began with intramuscular injection of HCG 10,000 IU. Oocyte puncture was then performed 36 hours after administration of HCG under guidance trans-vaginal ultrasonography. A maximum of 3 embryos were transferred on the fifth day after the puncture and the remaining embryos were frozen. Luteal phase support commenced on the day of embryo transfer in all the patients using $400 \mathrm{mg}$ twice daily progesterone vaginal suppositories (Cyclogest, Actavis, Devon, UK). Serum beta hCG levels were evaluated for all patients 14 to 16 days after embryo transfer to confirm biochemical pregnancy. Progestin support continued up to the end of the 12th weeks' gestation if the pregnancy was achieved. After embryo transfer, the following criteria were followed and evaluated: total dosage and duration of gonadotropin use, daily antagonist stimulation, endometrial thickness, and number of metaphase II oocyte, the number of transferred embryos, early miscarriage rate, implantation rate, and clinical pregnancy rate.

\section{Assisted reproduction techniques}

Oocytes were obtained from all three groups after $36 \mathrm{~h}$ under the guidance of sonography and after general anesthesia and the oocyte-cumulus, actually the COCs, were denuded immediately following puncture using hyaluronidase, and then the oocytes were incubated for two hours (maximum) before injection for 2 to 4 hours culture to remove cumulus oophorus and corona radiate by hyaluronidase solution. Then, the quality of the oocytes was evaluated and all mature oocytes (metaphase II) were fertilized by ICSI technique. and Commercial IVF culture media (Medi-Cult IVF media; Medi-Cult, Copenhagen, Denmark) was employed for embryo culture at $37^{\circ} \mathrm{C}$ with an atmosphere of $5 \% \mathrm{CO}_{2}$ and $20 \% \mathrm{O}_{2}$. Finally, fifth days after ICSI, the embryos were transferred into the uterine cavity. 
Assessment of embryos and zygotes

Zygotes were evaluated by the standards previously described by Tesarik (Wittemer et al., 2000). The morphological evaluation of the cleavage embryos was performed on day 2 and 3 based on the number of blastomeres, fragmentation, equality, mono nuclearity, and early compaction. The embryos that were classified as grade A or grade B (according to cleavage stage, blastomere size and shape, and fragmentation) were transferred. Patients in three groups were evaluated in terms of collected oocytes, MII oocytes, fertilized oocytes, the number of transferred oocytes, and chemical or clinical pregnancy.

\section{Statistical analysis}

For statistical analysis, results were presented as mean \pm standard deviation (SD) for quantitative variables, and were summarized using absolute frequencies and percentages for categorical variables. The normality of the data was analyzed using the Kolmogorov-Smirnoff test. The quantitative variables were also compared by ANOVA test or Kruskal-Wallis $\mathrm{H}$ test. For statistical analysis, SPSS (version 16.0) was used. P values $\leq 0.05$ were considered statistically significant.

\section{Results}

Comparing three groups with respect to baseline characteristics, including mean age, body mass index (BMI), the number of IVF procedure, the level of $\mathrm{AMH}, \mathrm{FSH}$, and LH hormones, Antral Follicle Counts (AFC), and duration of infertility, revealed no significant difference (Table 1). As shown in Table 2 and regarding the outcome of fertility inducing techniques, a significantly longer duration of $\mathrm{GnRH}$ antagonist treatment, higher dose of Gonal-f, lower number of collected, metaphase II, and fertilized oocytes, lower number of transferred embryos, and lower number of clinical pregnancy rate were observed in the control group as compared to two groups received GH. However, no significant was observed between two experimental groups. There was significant difference among three groups concerning the number of live births (one in A group, four in group $\mathrm{B}$, and no live birth in $\mathrm{C}$ group). The mean of endometrial thickness was significantly higher in group $\mathrm{B}$ after treatment as compared to other groups. There

Table 1. Baseline Characteristics of the Study Population

\begin{tabular}{lcccc}
\hline Index & Group A & Group B & Group C & P value \\
\hline Mean age, male & $38.91 \pm 5.01$ & $39.20 \pm 5.13$ & $39.17 \pm 4.31$ & 0.993 \\
Mean age, female & $33.80 \pm 4.66$ & $33.91 \pm 4.76$ & $33.91 \pm 4.49$ & 0.964 \\
BMI, kg/m & $26.43 \pm 3.24$ & $26.60 \pm 3.07$ & $26.63 \pm 3.07$ & 0.959 \\
Number of IVF & $1.37 \pm 0.81$ & $1.34 \pm 0.80$ & $1.34 \pm 0.80$ & 0.979 \\
Level of AMH & $0.91 \pm 0.32$ & $0.85 \pm 0.28$ & $0.89 \pm 0.25$ & 0.746 \\
Level of FSH & $7.05 \pm 1.74$ & $7.30 \pm 1.48$ & $7.14 \pm 1.68$ & 0.81 \\
Level of LH & $6.02 \pm 1.38$ & $6.07 \pm 1.28$ & $6.03 \pm 1.21$ & 0.978 \\
Antral follicle counts & $6.54 \pm 1.74$ & $6.60 \pm 1.68$ & $6.66 \pm 1.69$ & 0.961 \\
Duration of infertility, y & $3.71 \pm 1.63$ & $3.69 \pm 1.61$ & $3.16 \pm 1.62$ & 0.997 \\
\hline
\end{tabular}

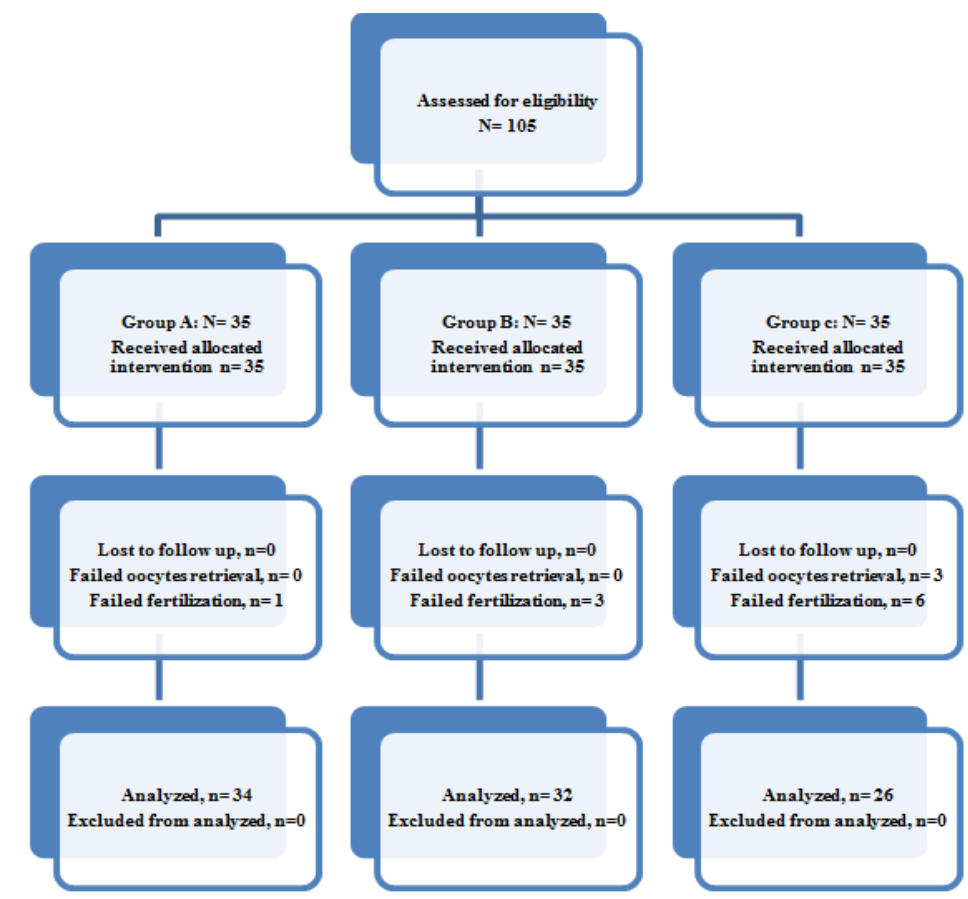

Figure 1. CONSORT Flow Diagram of the Progress of Pparticipants of the Randomized Trial 
Table 2. The Therapeutic Outcome in the Study Population

\begin{tabular}{lcccc}
\hline Index & Group A & Group B & Group C & P value \\
\hline Duration of gonadotropin (Gonal-f) treatment, d & $10.23 \pm 1.03$ & $10.37 \pm 1.08$ & $13.29 \pm 1.38$ & 0.051 \\
Duration of GnRH antagonist treatment, d & $2.20 \pm 0.58$ & $2.11 \pm 0.32$ & $3.89 \pm 0.90$ & $<0.001$ \\
Total doses of Gonal-f, IU & $3098.57 \pm 510.26$ & $3140.00 \pm 490.47$ & $4662.86 \pm 213.61$ & $<0.001$ \\
Endothelial thickness, mm & $10.51 \pm 0.81$ & $11.14 \pm 0.77$ & $10.54 \pm 0.85$ & 0.002 \\
No. of collected oocytes & $7.14 \pm 2.03$ & $7.29 \pm 2.16$ & $5.17 \pm 1.82$ & $<0.001$ \\
No. of MII oocytes & $6.09 \pm 1.65$ & $6.09 \pm 2.00$ & $3.46 \pm 2.09$ & $<0.001$ \\
No. of fertilized oocytes & $5.00 \pm 1.96$ & $5.43 \pm 2.48$ & $2.17 \pm 1.90$ & $<0.001$ \\
No. of transferred embryos & $2.49 \pm 0.66$ & $2.40 \pm 0.88$ & $1.63 \pm 1.39$ & $<0.001$ \\
Chemical pregnancy, \% & $9(25.7 \%)$ & $10(28.6 \%)$ & $3(8.6 \%)$ & 0.084 \\
Clinical pregnancy, \% & $5(14.3 \%)$ & $8(22.9 \%)$ & $1(2.9 \%)$ & 0.047 \\
No. of live birth, \% & $1(2.9 \%)$ & $4(11.4 \%)$ & $0(0.0 \%)$ & 0.357 \\
\hline
\end{tabular}

was no significant difference concerning the frequency of successful chemical pregnancy and also the number of live births, but clinical pregnancy was significantly higher than two other groups.

\section{Discussion}

Our study findings revealed that co-treatment with $\mathrm{GH}$ in PORs could improve the rate of clinical pregnancy and the number of collected oocytes (metaphase II), fertilized oocytes, and transferred embryos; however, we did not find the effect of this treatment on chemical pregnancy and the number of live births. A recent systematic review and meta-analysis by Li et al., (2017) demonstrated that co-treatment with $\mathrm{GH}$ in controlled ovarian stimulation cycles could improve clinical pregnancy rate, live birth rate, the number of collected oocytes, and the number of MII oocytes, but they reported no significant difference between experimental and control groups in terms of implantation and fertilization rates. In total, adding GH on a routine regimen for ovary stimulation can increase the likelihood of clinical pregnancy and also can mature fertilized oocytes. As previously demonstrated, GH has a critical role in ovarian functional status because of its effect on granulose cells growth through inducing secretion of IGF-1. Growth factors are polypeptides that modulate cell proliferation and differentiation operating through binding to specific cell membrane receptors. They act locally and function in paracrine and autocrine modes. There are multiple growth factors, and most cells contain multiple receptors for the various growth factors. Insulinlike growth factors are peptides that have structural and functional similarity to insulin and can mediate growth hormone action (Laron, 2001). IGF-I mediates the growth-promoting actions of growth hormone. The majority of circulating IGF-I is derived from growth hormone- dependent synthesis in liver. It is believed to be important in fetal growth and development. Both IGFs induce the expression of cellular genes responsible for cellular proliferation and differentiation (Grimberg and Cohen, 2000). IGF-I has been demonstrated to stimulate the following events in ovarian theca and granuosa cells: DNA synthesis, steroidogenesis, aromarase activity, LH receptor synthesis, and inhibin secretion. IGF-II stimulates granulosa mitosis. In human ovarian cells, IGF-I, in synergy with FSH, stimulates protein synthesis and steroidogenesis. After LH receptors presence, IGF-I enhances LH- induced progesterone synthesis and stimulates proliferation of granulosa-luteal cells. IGF-I, in synergy with FSH, is very influential in stimulating aromatase activity in preovulatory follicles. Thus, IGF-I can be involved in both estradiol and progesterone synthesis (Zhou et al., 2013)

In other word, the existence of $\mathrm{GH}$ is essential in follicle development and ovarian steroidogenesis. This effective role has also been indicated in some animal studies, yielding that administration of GH and IGF-1 can stimulate oocyte maturation (Kiapekou et al., 2005). However, in some earlier meta- analysis, no significant difference was revealed between GH group and control group in terms of clinical pregnancy rate, which was not consistent with the findings of the present trial (Jeve and Bhandari, 2016). More interestingly, the baseline hormonal and fertilizing conditions of the patients as well as the dose of $\mathrm{GH}$ also influence the beneficial effects of GH in patients scheduling for IVF/IUI. Some studies have shown that low-dose GH supplementation increase clinical pregnancy rate in PORs undergoing IVF (Lattes et al., 2015). Some others reported high efficacy of GH in PORs with repeated IVF failures (Hazout et al., 2009). Some investigators have suggested the baseline GH deficiency as a necessary condition for the effectiveness of GH in those women who are candidates for IVF (Rajesh et al., 2007). Another finding is that exogenous GH may have multi potential effects on ovarian stimulation. As indicated by Gregoraszczuk et al., (2000), GH addition can stimulate both estradiol and progesterone secretion from large pre-ovulatory follicles. However, Tapanainen et al., (1992) has shown lower serum E2 level in patients received $\mathrm{GH}$ than in those in placebo group. These discrepancies over the beneficial effects of GH on PORs might be due to administration of different dosages of $\mathrm{GH}$ in various studies, and the different definition of POR or due to difference in studied subgroups. Regarding dosage of GH, a range of 4 IU to 12 IU was considered in 2 to 3 subgroups of PORs in previous studies. These differences led to high heterogeneity between findings of studies according to a recent meta-analysis ( $\mathrm{Li}$ et al., 
2017). Thus, for achieving valid results on benefits of GH administration for ROPs who are candidates for IVF/IUI, further trials considering different timings and doses of GH should be performed.

In conclusion this study, we found that GH played an important role in the recruitment of the dominant follicle from its cohort and could enhance follicular survival and the cell proliferation, leading to high-quality embryos. We also found that the administration of GH (for 5 to 20 days) could considerably intensify the ovarian response in PORs who were candidates for IVF - IUI. Moreover, long-term adminstration of GH (from the third day of the previous cycle) improved implantation and clinical pregnancy outcomes due its supposed effect on the endometrial thickening.

\section{Limitations}

We did not study the safety of long-term administration of GH on mothers and their children; however, no adverse effects was reported during the course of the study.

\section{Acknowledgments}

This study was performed at the Shariati Hospital of Tehran University of Medical Sciences.

\section{References}

Gregoraszczuk EL, Bylica A, Gertler A (2000). Response of porcine theca and granulosa cells to GH during short-term in vitro culture. Anim Reprod Sci, 58, 113-25.

Grimberg A, Cohen P (2000). Role of insulin-like growth factors and their binding proteins in growth control and carcinogenesis. J Cell Physiol, 183, 1-9.

Hamdine O, Eijkemans MJ, Lentjes EW, et al (2015). Ovarian response prediction in $\mathrm{GnRH}$ antagonist treatment for IVF uses anti-Müllerian hormone. Hum Reprod, 30, 170-8.

Hazout A, Junca A, Menezo Y (2009). Effect of growth hormone on oocyte competence in patients with multiple IVF failures. Reprod Biomed Online, 18, 664-70.

Jeve YB, Bhandari HM (2016). Effective treatment protocol for poor ovarian response: a systematic review and metaanalysis. J Hum Reprod Sci, 9, 70-81.

Kiapekou E, Loutradis D, Drakakis P, et al (2005). Effects of $\mathrm{GH}$ and IGF-I on the in vitro maturation of mouse oocytes. Hormones, 4, 155-60.

Kolibianakis EM, Venetis CA, Diedrich K, et al (2009). Addition of growth hormone to gonadotrophins in ovarian stimulation of poor responders treated by in-vitro fertilization: a systematic review and meta-analysis. Hum Reprod Update, 15, 613-22.

Kucuk T, Kozinoglu H, Kaba A (2008). Growth hormone co treatment within a $\mathrm{GnRH}$ agonist long protocol in patients with poor ovarian response: a prospective, randomized, clinical trial. J Assist Reprod Genet, 25, 123-7.

Laron Z (2001). Insulin-like growth factor 1 (IGF-1): a growth hormone. Mol Pathol, 54, 311-6.

Lattes K, Brassesco M, Gomez M, et al (2015). Low-dose growth hormone supplementation increases clinical pregnancy rate in poor responders undergoing in vitro fertilization. Gynecol Endocrinol, 31, 565-8.

Li XL, Wang L, Lv F, et al (2017). The influence of different growth hormone addition, protocols to poor ovarian responders on clinical outcomes in controlled ovarian stimulation cycles: A systematic review and meta-analysis. Medicine (Baltimore), 96, e6443.

Lucy MC (2000). Regulation of ovarian follicular growth by somatotropin and insulin-like growth factors in cattle. J Dairy Sci, 83, 1635-47.

Lucy MC (2011). Growth hormone regulation of follicular growth. Reprod Fertil Dev, 24, 19-28.

Mendoza C, Ruiz-Requena E, Ortega E, et al (2002). Follicular fluid markers of oocyte developmental potential. Hum Reprod, 17, 1017-22.

Oehninger S (2011). Poor responders in vitro fertilization (IVF) therapy: the challenge continues. Facts Views Vis Obgyn, 3, 101-8.

Rajesh H, Yong YY, Zhu M (2007). Growth hormone deficiency and supplementation at in-vitro fertilization. Singapore MED J, 48, 514-8.

Sooyoung K, Younji L, Sanghoon L (2018). Ovarian tissue cryopreservation and transplantation in patients with cancer. Obstet Gynecol Sci, 61, 431-42.

Tapanainen J, Martikainen H, Voutilainen R, et al (1992). Effect of growth hormone administration on human ovarian function and steroidogenic gene expression in granulosaluteal cells. Fertile Sterile, 58, 726 -32.

Tesarik J, Rienzi L, Ubaldi F, et al (2002). Use of a modified intra cytoplasmic sperm injection technique to overcome sperm-borne and oocyte-borne oocyte activation failures. Fertile Sterile, 78, 619-24.

Venetis CA, Kolibianakis EM, Tarlatzi TB, et al (2010). Evidence-based management of poor ovarian response. Ann N Y Acad Sci, 1205, 199-206.

Wittemer C, Bettahar-Lebugle K, Ohl J, et al (2000). Zygote evaluation: an efficient tool for embryo selection. Hum Reprod, 15, 2591-7.

Zhou P, Baumgarten SC, Wu Y, et al (2013). IGF-I signaling is essential for FSH stimulation of AKT and steroidogenic genes in granulosa cells. Mol Endocrinol, 27, 511-23.

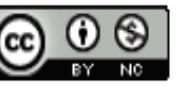

This work is licensed under a Creative Commons AttributionNon Commercial 4.0 International License. 